\title{
Complementary NMR-based metabolomics approaches for glioma biomarker identification in a Drosophila melanogaster model
}

Marion Maravat ${ }^{1}$, Marylène Bertrand ${ }^{2}$, Céline Landon ${ }^{2}$, Franck Fayon ${ }^{1}$, Séverine Morisset-Lopez ${ }^{2}$, Vincent Sarou-Kanian ${ }^{1}$, Martine Decoville ${ }^{2 *}$

${ }^{1}$ CNRS, CEMHTI UPR3079, Université d'Orléans, F-45071, Orléans, France

${ }^{2}$ CNRS, CBM UPR4301, Université d'Orléans, F-45071, Orléans, France

* Corresponding author : martine.decoville@cnrs-orleans.fr

\section{Supporting Information}

Figure S1 : Comparison of the mean solution-state NMR spectra of control and glioma samples.

Figure S2 : Comparison of the mean HR-MAS spectra of control and glioma samples.

Table S1 : Solution-state ${ }^{1} \mathrm{H}$ NMR and ${ }^{1} \mathrm{H}$ HR-MAS signals of the identified metabolites discriminating glioma brains from control samples and the impacted metabolic pathways.

Table S2 : Performance measurement of the characteristic bucket(s) of each metabolite.

Figure S3 : Biomarker analysis on a group of four selected metabolites (glucose, myo-inositol, sarcosine and glycine) from the solution-state NMR results.

Figure S4 : Biomarker analysis on a group of four selected metabolites (proline, myo-inositol, acetate and glucose) from the HR-MAS results. 
Figure S1: Comparison of the mean solution-state ${ }^{\mathbf{1}} \mathrm{H}$ NMR spectra of control and glioma samples. Each spectrum is an average of all the spectra obtained for each condition. Blue: glioma; green: control; (a-c): different spectral regions.

(a)

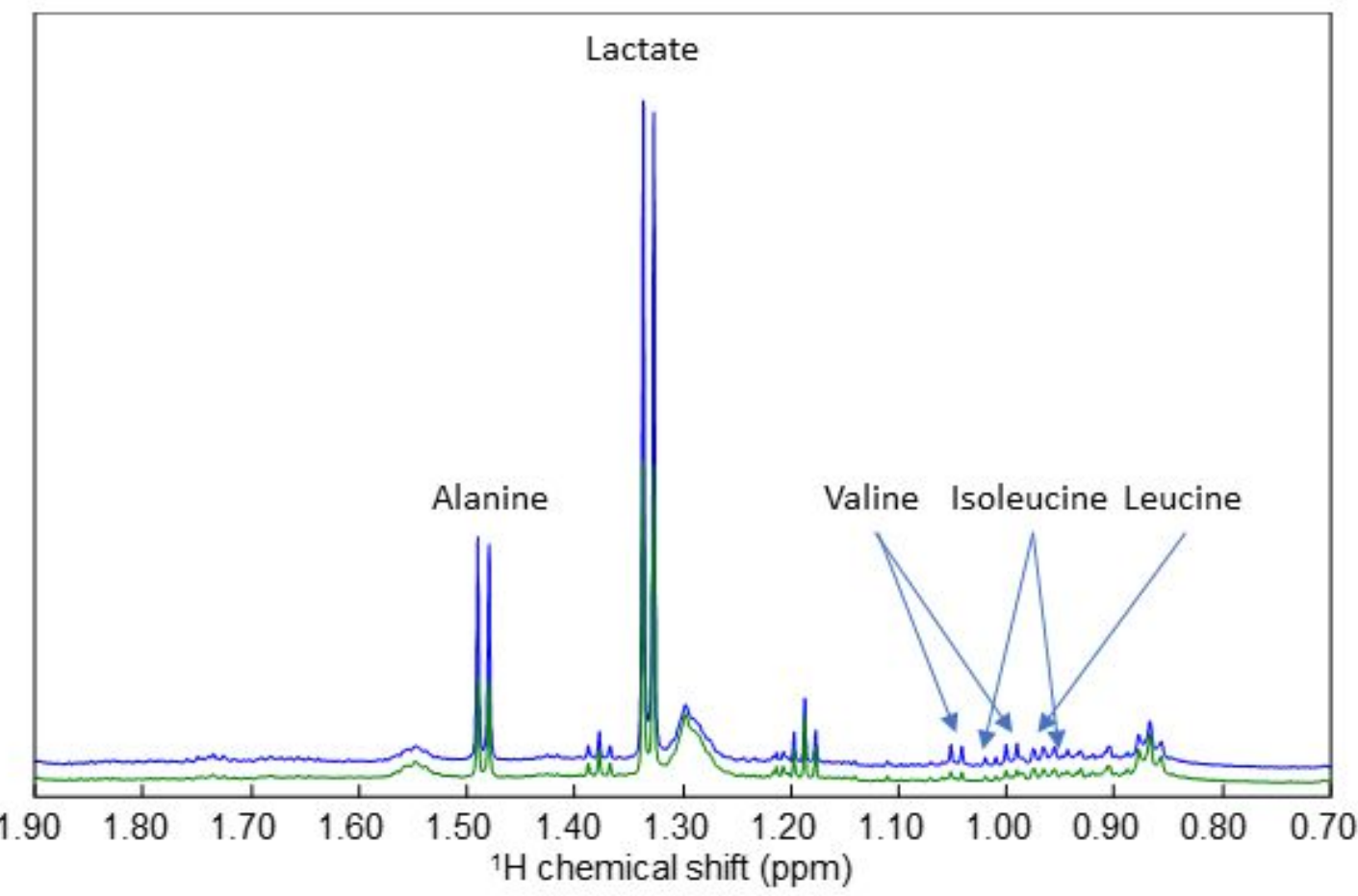

(b)

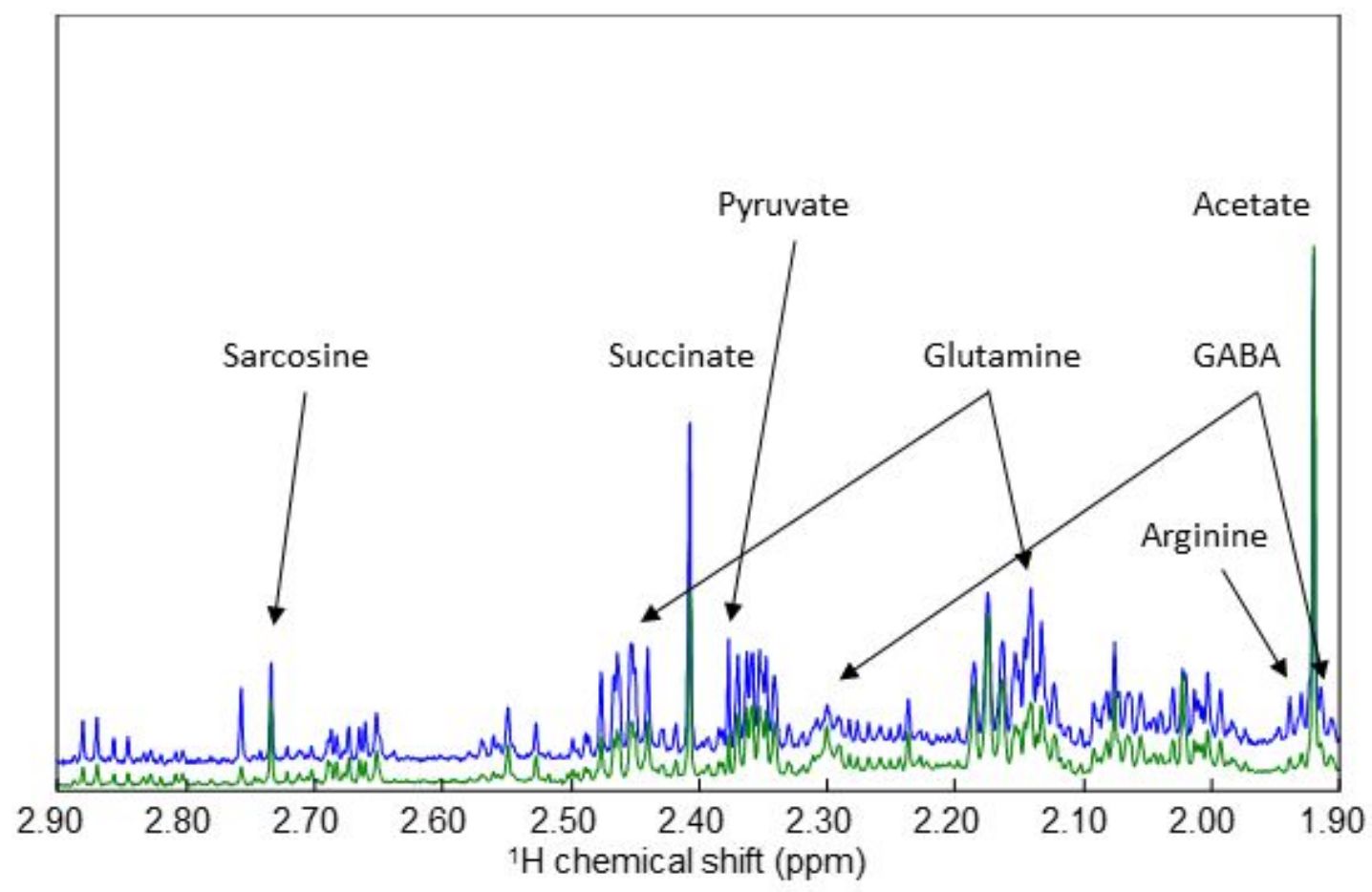


(c)

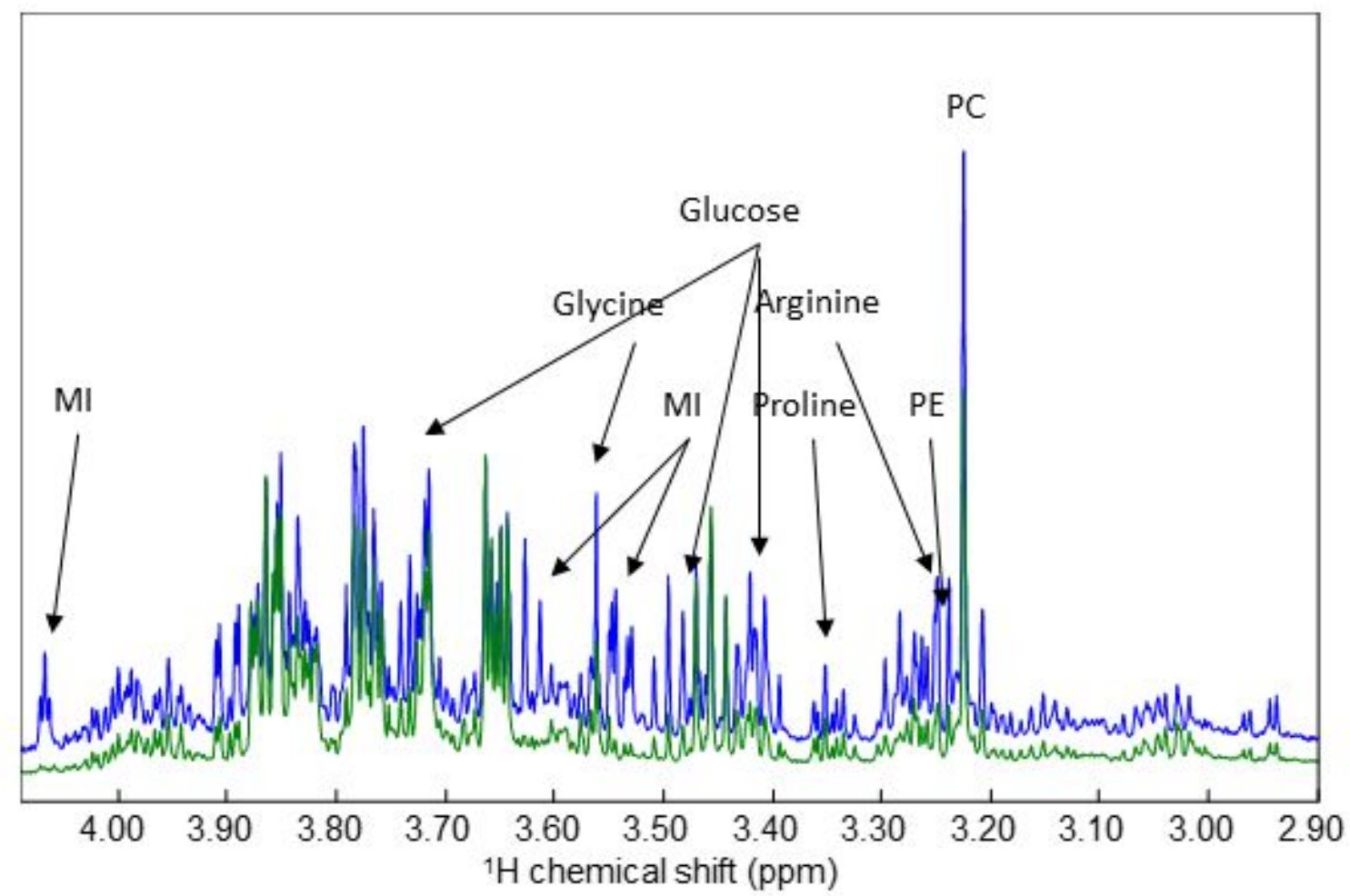


Figure S2: Comparison of the mean solution-state ${ }^{1} \mathrm{H}$ HR-MAS spectra of control and glioma samples. Each spectrum is an average of all the spectra obtained for each condition. Blue: glioma; green: control; (a-b): different spectral regions.

(a)

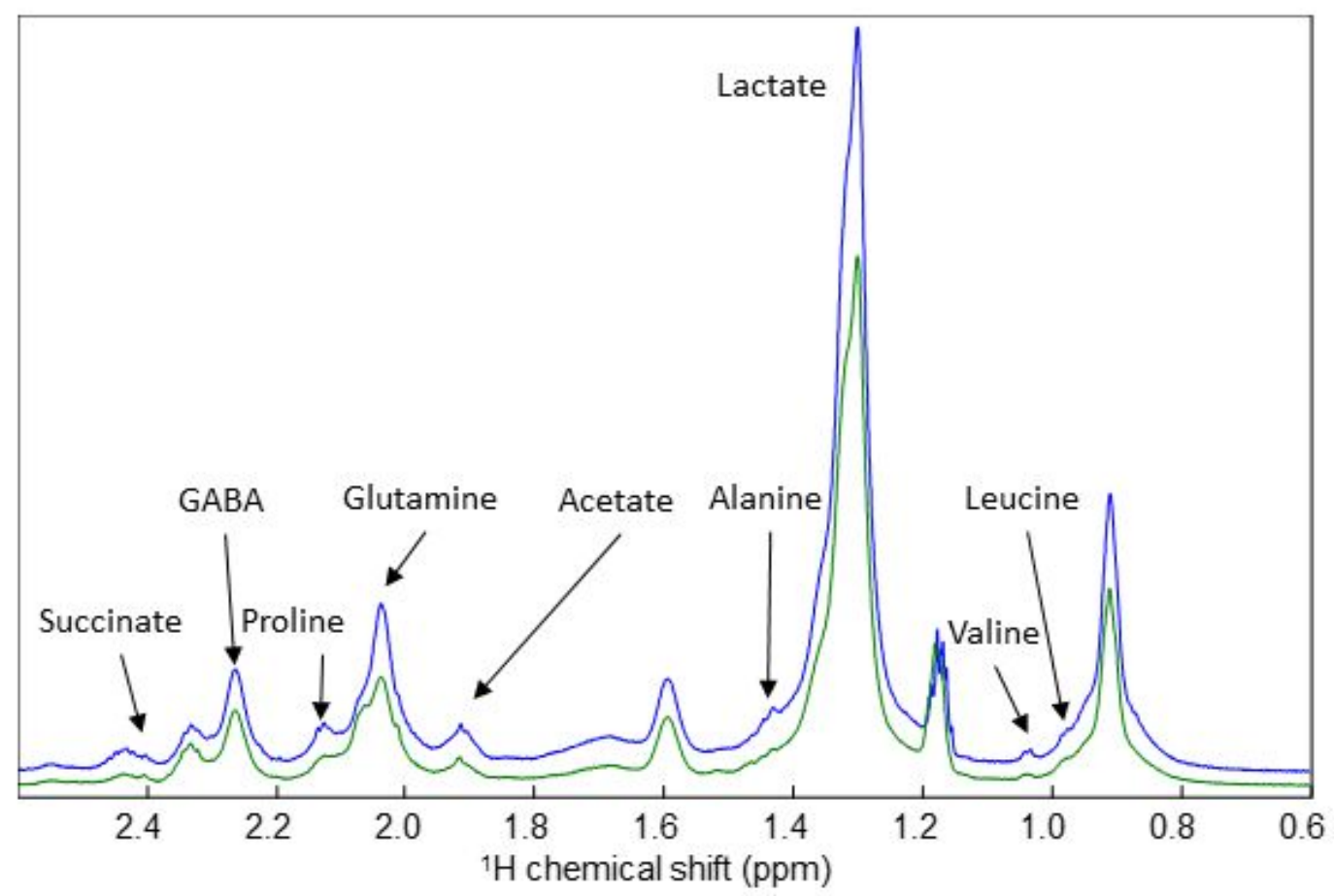

(b)

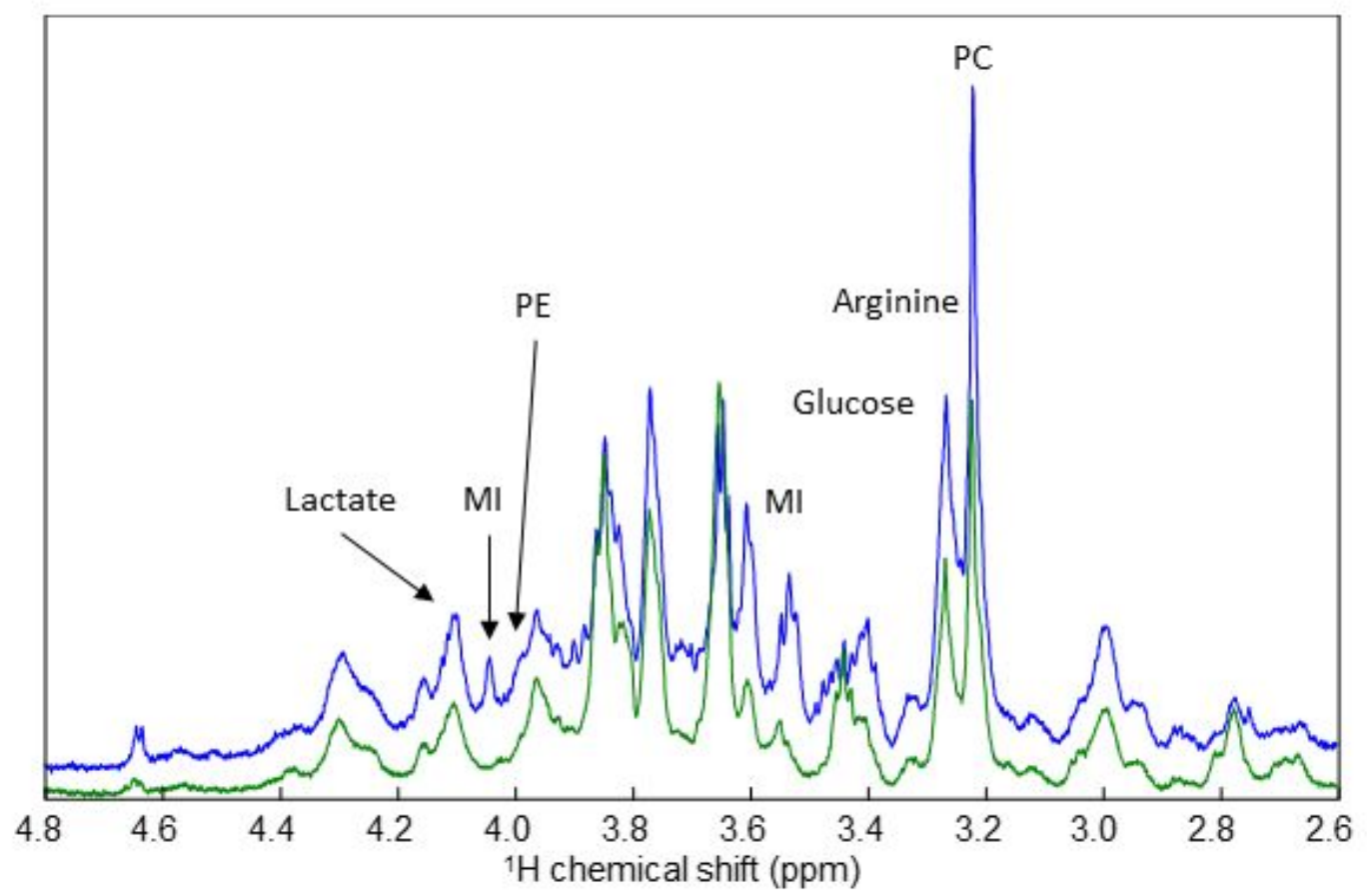




\begin{tabular}{|c|c|c|c|c|c|c|c|}
\hline & \multirow{2}{*}{\multicolumn{3}{|c|}{ Discriminant metabolites }} & \multirow{2}{*}{\multicolumn{2}{|c|}{ Solution-state NMR }} & & \\
\hline & & & & & & \multicolumn{2}{|c|}{ HR-MAS } \\
\hline & Metabolite & Group & $\begin{array}{l}\text { H chemical } \\
\text { shift (ppm) }\end{array}$ & Levels & p value & Levels & $p$ value \\
\hline 1 & Acetate & $\mathrm{CH}_{3}$ & 1,92 & $\pi$ & $* *$ & $\pi$ & $* * *$ \\
\hline \multirow[t]{2}{*}{2} & Alanine & $\mathrm{CH}_{3}$ & 1,48 & $\pi$ & $* * *$ & $\pi$ & $*$ \\
\hline & & $\mathrm{CH}$ & $3,78^{\#}$ & $\pi$ & $* * *$ & & \\
\hline \multirow[t]{3}{*}{3} & Arginine & $\mathrm{CH}_{2}$ & $1,6-1,7$ & $\pi$ & $* * *$ & $\pi$ & $* *$ \\
\hline & & $\mathrm{CH}_{2}$ & $1,90-1,93^{\#}$ & $\pi$ & $* * *$ & $\pi$ & $* *$ \\
\hline & & $\mathrm{CH}_{2}$ & $3,24^{\#}$ & $\pi$ & $* * *$ & $\pi$ & $* *$ \\
\hline \multirow[t]{3}{*}{4} & Benzoate & $\mathrm{CH}$ & 7,49 & $y$ & $* * *$ & & \\
\hline & & $\mathrm{CH}$ & 7,56 & $y$ & $* * *$ & & \\
\hline & & $\mathrm{CH}$ & 7,88 & $y$ & $* * *$ & & \\
\hline \multirow[t]{3}{*}{5} & GABA & $\mathrm{CH}_{2}$ & 1,86 & $y$ & $* *$ & & \\
\hline & & $\mathrm{CH}_{2}$ & 2,29 & $y$ & $* * *$ & $y$ & $* *$ \\
\hline & & $\mathrm{CH}_{2}$ & 3,02 & $y$ & $* *$ & & \\
\hline \multirow[t]{7}{*}{6} & Glucose & $\mathrm{CH}_{2}$ & $3,25^{\#}$ & $\pi$ & $* * *$ & $\pi$ & $* *$ \\
\hline & & $\mathrm{CH}$ & $3,41^{\#}$ & $\pi$ & $* * *$ & & \\
\hline & & $\mathrm{CH}$ & $3,47-3,50$ & $\pi$ & $* * *$ & & \\
\hline & & $\mathrm{CH}$ & $3,70-3,74^{\#}$ & $\pi$ & $* * *$ & & \\
\hline & & $\mathrm{CH}$ & $3,89-3,91$ & $\pi$ & $* * *$ & & \\
\hline & & $\mathrm{CH}$ & $4,65-4,66$ & $\pi$ & $* * *$ & & \\
\hline & & $\mathrm{CH}$ & $5,24-5,25$ & $\pi$ & $* * *$ & & \\
\hline \multirow[t]{3}{*}{7} & Glutamine & $\mathrm{CH}_{2}$ & 2,14 & $\pi$ & $* * *$ & $\pi$ & $*$ \\
\hline & & $\mathrm{CH}_{2}$ & $2,44-2,48$ & $\pi$ & $* * *$ & $\pi$ & $*$ \\
\hline & & $\mathrm{CH}_{2}$ & $3,75^{\#}$ & $\pi$ & $* * *$ & & \\
\hline 8 & Glycine & $\mathrm{CH}_{2}$ & 3,56 & $\pi$ & $* * *$ & & \\
\hline \multirow[t]{4}{*}{9} & Isoleucine & $\mathrm{CH}_{3}$ & $0,93^{\#}$ & $y$ & $* *$ & & \\
\hline & & $\mathrm{CH}_{3}$ & 1,02 & $y$ & $* * *$ & & \\
\hline & & $\mathrm{CH}_{2}$ & $1,45-1,47^{\#}$ & $y$ & $* * *$ & & \\
\hline & & $\mathrm{CH}$ & $1,97^{\#}$ & $y$ & $* * *$ & & \\
\hline \multirow[t]{2}{*}{10} & Lactate & $\mathrm{CH}_{3}$ & $1,33-1,34$ & $\pi$ & $* * *$ & & \\
\hline & & $\mathrm{CH}$ & $4,10-4,13$ & $\pi$ & $* * *$ & $\pi$ & $*$ \\
\hline \multirow[t]{2}{*}{11} & Leucine & $2 \mathrm{CH}_{3}$ & $0,95-0,97^{\#}$ & $\pi$ & $* *$ & $\pi$ & $* *$ \\
\hline & & $\mathrm{CH}_{2}$ & $1,66-1,73^{\#}$ & & & $\pi$ & $* *$ \\
\hline \multirow[t]{4}{*}{12} & Myoinositol & $\mathrm{CH}$ & 3,27 & $\pi$ & $* * *$ & $\pi$ & $* *$ \\
\hline & & $2 \mathrm{CH}$ & $3,52-3,53$ & $\pi$ & $* * *$ & $\pi$ & $* * *$ \\
\hline & & $2 \mathrm{CH}$ & $3,61-3,63$ & $\pi$ & $* * *$ & $\pi$ & $* * *$ \\
\hline & & $\mathrm{CH}$ & 4,07 & $\pi$ & $* * *$ & & \\
\hline \multirow[t]{3}{*}{13} & PC & $\mathrm{CH}_{3}$ & 3,21 & & & $\pi$ & $* *$ \\
\hline & & $\mathrm{CH}_{2}$ & 3,58 & & & $\pi$ & $*$ \\
\hline & & $\mathrm{CH}_{2}$ & 4,15 & & & $\pi$ & $* * *$ \\
\hline \multirow[t]{2}{*}{14} & PE & $\mathrm{CH}_{2}$ & $3,20-3,22^{\#}$ & $\pi$ & $* *$ & $\pi$ & $* *$ \\
\hline & & $\mathrm{CH}_{2}$ & $3,98-4,00^{\#}$ & $\pi$ & $* *$ & $\pi$ & $* *$ \\
\hline \multirow[t]{3}{*}{15} & Proline & $\mathrm{CH}_{2}$ & $1,98-2,10^{\#}$ & & & $y$ & $* * *$ \\
\hline & & $\mathrm{CH}_{2}$ & $2,32-2,36^{\#}$ & & & $y$ & $* *$ \\
\hline & & $\mathrm{CH}_{2}$ & $3,32-3,43^{\#}$ & $y$ & $* *$ & & \\
\hline 16 & Pyruvate & $\mathrm{CH}_{3}$ & 2,37 & $\pi$ & $* * *$ & & \\
\hline 17 & Sarcosine & $\mathrm{CH}_{3}$ & 2,75 & $\pi$ & $* * *$ & & \\
\hline & & $\mathrm{CH}_{2}$ & $3,61^{\#}$ & $\pi$ & $* * *$ & & \\
\hline 18 & Succinate & $2 \mathrm{CH}_{2}$ & 2,40 & $\pi$ & $* * *$ & $\pi$ & $*$ \\
\hline 19 & Tyrosine & $\mathrm{CH}_{2}$ & $3,05^{\#}$ & $\pi$ & $* * *$ & & \\
\hline & & $\mathrm{CH}_{2}$ & $3,2^{\#}$ & $\pi$ & $* *$ & & \\
\hline & & $\mathrm{CH}_{2}$ & $3,92-3,94^{\#}$ & $\pi$ & $* *$ & & \\
\hline & & $2 \mathrm{CH}$ & 6,91 & $\pi$ & $* *$ & & \\
\hline & & $2 \mathrm{CH}$ & $7,2^{\#}$ & $\pi$ & $* * *$ & & \\
\hline 20 & Valine & $\mathrm{CH}_{3}$ & $0,99-1,00$ & $\pi$ & $*$ & $\pi$ & $*$ \\
\hline & & $\mathrm{CH}_{3}$ & $1,03-1,04$ & $\pi$ & $* *$ & $\pi$ & $*$ \\
\hline
\end{tabular}

Table S1 : Solution-state ${ }^{1} \mathrm{H}$ NMR and ${ }^{1} \mathrm{H}$ HR-MAS signals of the identified metabolites discriminating glioma brains from control samples and the impacted metabolic pathways. $\nearrow$ : increased relative levels and $\searrow$ : decreased relative levels in the glioma samples compared to the controls. * p value $<0.05$, ** p value $<0.01$, *** p value $<0.001$. \# one or several peaks of multiplet overlapped. 


\begin{tabular}{lcccccr}
\cline { 2 - 7 } & \multicolumn{2}{c}{ Solution-state NMR } & \multicolumn{3}{c}{ HR-MAS } \\
\cline { 2 - 7 } Name & Chemical shift & AUC & t-test & Chemical shift & AUC & t-test \\
\hline Acetate & $1.92 \mathrm{ppm}$ & 0,821 & $2,97 \mathrm{E}-03$ & $1.92 \mathrm{ppm}$ & 0,929 & $8,87 \mathrm{E}-06$ \\
Alanine & $1.49 \mathrm{ppm}$ & 0,996 & $5,94 \mathrm{E}-11$ & $1.44 \mathrm{ppm}$ & 0,835 & $1,68 \mathrm{E}-03$ \\
Arginine & $3.24 \mathrm{ppm}$ & 0,988 & $1,41 \mathrm{E}-08$ & $1.93 \mathrm{ppm}$ & 0,882 & $7,63 \mathrm{E}-05$ \\
GABA & $2.30 \mathrm{ppm}$ & 0,842 & $1,38 \mathrm{E}-03$ & $2.30 \mathrm{ppm}$ & 0,761 & $3,83 \mathrm{E}-02$ \\
Glucose & $3.26 \mathrm{ppm}$ & 1,000 & $6,09 \mathrm{E}-17$ & $3.24 \mathrm{ppm}$ & 0,898 & $6,73 \mathrm{E}-05$ \\
Glutamine & $2.46 \mathrm{ppm}$ & 0,913 & $2,85 \mathrm{E}-05$ & $2.14 \mathrm{ppm}$ & 0,757 & $6,49 \mathrm{E}-03$ \\
Glycine & $3.57 \mathrm{ppm}$ & 1,000 & $1,64 \mathrm{E}-10$ & & & \\
Isoleucine & $1.46 \mathrm{ppm}$ & 0,875 & $3,40 \mathrm{E}-04$ & & & \\
Lactate & $1.33 \mathrm{ppm}$ & 0,904 & $5,13 \mathrm{E}-05$ & & & \\
Lactate & $4.13 \mathrm{ppm}$ & 0,971 & $3,76 \mathrm{E}-07$ & $4.12 \mathrm{ppm}$ & 0,749 & $3,40 \mathrm{E}-02$ \\
Leucine & $0.97 \mathrm{ppm}$ & 0,883 & $1,76 \mathrm{E}-04$ & $0.94 \mathrm{ppm}$ & 0,831 & $2,03 \mathrm{E}-03$ \\
Myo-inositol & $3.27 \mathrm{ppm}$ & 0,983 & $1,96 \mathrm{E}-09$ & $3.27 \mathrm{ppm}$ & 0,918 & $1,15 \mathrm{E}-05$ \\
Myo-inositol & $3.54 \mathrm{ppm}$ & 1,000 & $1,24 \mathrm{E}-16$ & $3.52 \mathrm{ppm}$ & 0,969 & $4,91 \mathrm{E}-09$ \\
PC & & & & $3.21 \mathrm{ppm}$ & 0,800 & $3,93 \mathrm{E}-03$ \\
PE & $4.00 \mathrm{ppm}$ & 0,988 & $1,01 \mathrm{E}-08$ & $3.99 \mathrm{ppm}$ & 0,871 & $1,97 \mathrm{E}-04$ \\
Proline & $3.32 \mathrm{ppm}$ & 0,850 & $4,70 \mathrm{E}-03$ & $2.06 \mathrm{ppm}$ & 0,976 & $3,68 \mathrm{E}-10$ \\
Proline & & & & $2.33 \mathrm{ppm}$ & 0,886 & $7,80 \mathrm{E}-05$ \\
Pyruvate & $2.38 \mathrm{ppm}$ & 0,992 & $3,81 \mathrm{E}-10$ & & & \\
Sarcosine & $2.76 \mathrm{ppm}$ & 1,000 & $5,94 \mathrm{E}-11$ & & & \\
Succinate & $2.41 \mathrm{ppm}$ & 0,879 & $5,77 \mathrm{E}-05$ & $2.41 \mathrm{ppm}$ & 0,753 & $9,78 \mathrm{E}-03$ \\
Tyrosine & $7.21 \mathrm{ppm}$ & 0,950 & $1,44 \mathrm{E}-07$ & & & \\
Valine & $1.05 \mathrm{ppm}$ & 0,900 & $1,22 \mathrm{E}-05$ & $1.03 \mathrm{ppm}$ & 0,859 & $7,23 \mathrm{E}-05$ \\
\hline
\end{tabular}

Table S2 : Performance measurement of the characteristic bucket(s) of each metabolite. AUC and $t$-test are the factors used to determine the ability to separate glioma samples from controls. 

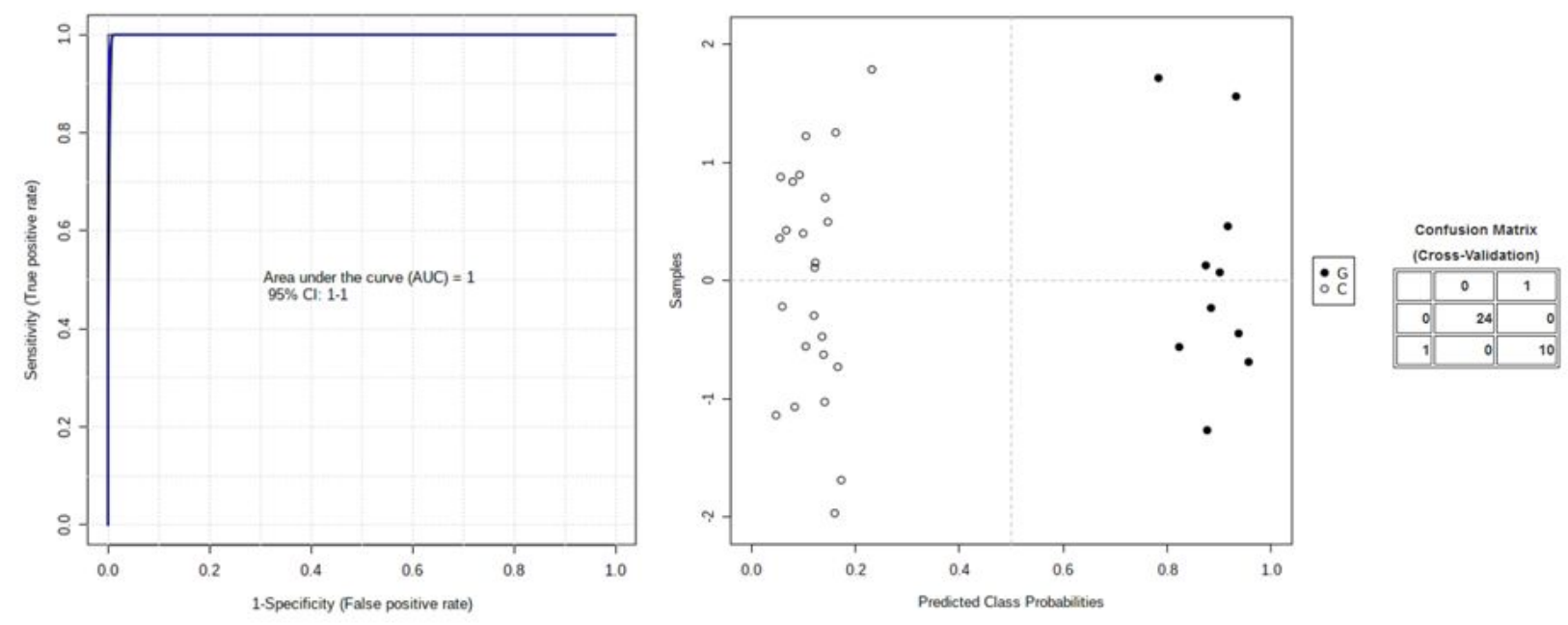

Figure S3 : Biomarker analysis on a group of four selected metabolites (glucose, myo-inositol, sarcosine and glycine) from the solution-state NMR results.

Left: smooth ROC curve performed with 100 cross validations (CV), the results were averaged to generate the plot. Right: average of predicted class probabilities of each sample across the 100 cross-validations (with linear SVM algorithm) and confusion matrix with the classification of the glioma samples $\mathrm{G}$ and the control samples $\mathrm{C}$.
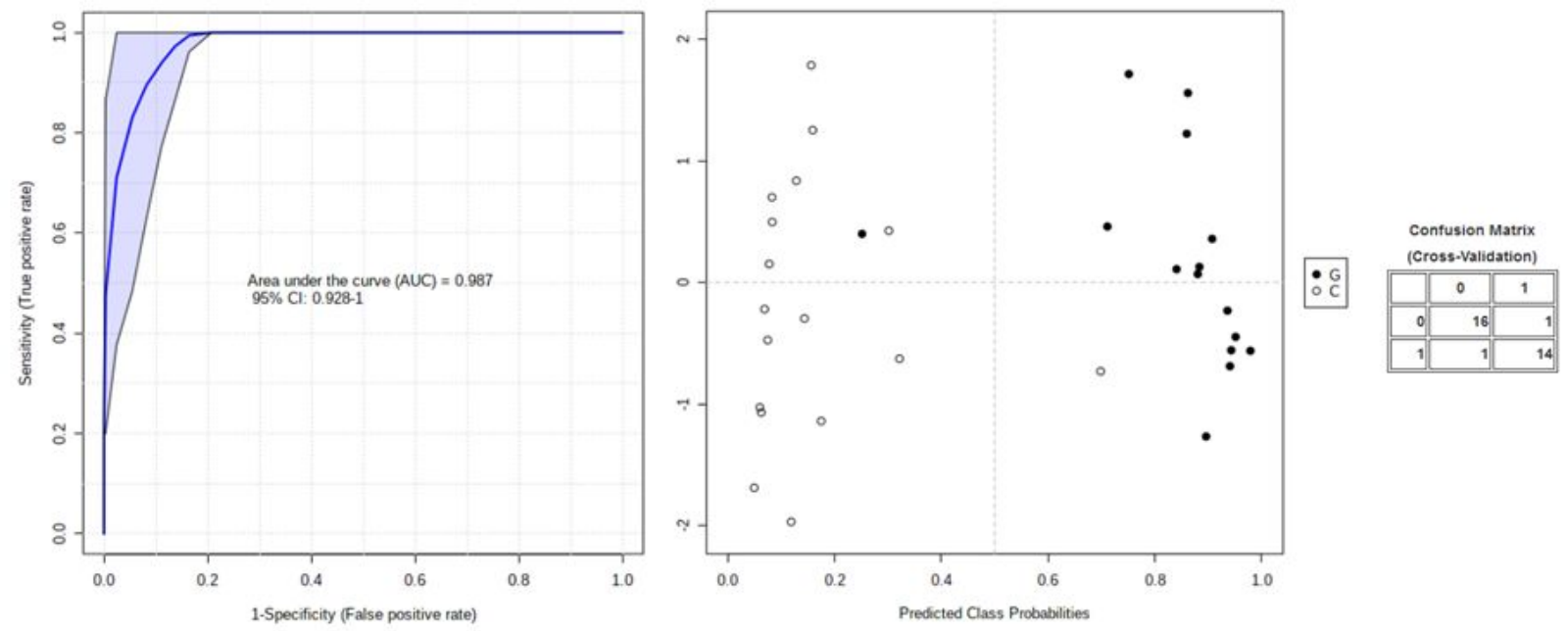

Figure S4 : Biomarker analysis on a group of four selected metabolites (proline, myo-inositol, acetate and glucose) from the HR-MAS results.

Left: smooth ROC curve performed with 100 cross validations (CV), the results were averaged to generate the plot. Right: average of predicted class probabilities of each sample across the 100 cross-validations (with linear SVM algorithm) and confusion matrix with the classification of the glioma samples $\mathrm{G}$ and the control samples C. Two samples are not sorted in the right class. 